\title{
Computer-Aided Modeling of Pentachlorophenol 4-Monooxygenase and Site-Directed Mutagenesis of Its Active Site
}

\author{
Takashi Nakamura, ${ }^{a}$ Takayuki Motoyama, ${ }^{a}$ Takatsugu Hirokawa, ${ }^{b}$ Shuichi Hirono, ${ }^{c}$ and \\ Isamu YAMAGUCHI ${ }^{*, a}$ \\ ${ }^{a}$ Laboratory for Remediation Research, Environmental Plant Research Group, Plant Science Center, RIKEN Institute; \\ 1-7-22 Suehiro, Tsurumi-ku, Yokohama, Kanagawa 230-0045, Japan: ${ }^{b}$ Computational Biology Research Center (CBRC), \\ National Institute of Advanced Industrial Science and Technology (AIST); 2-41-6 Aomi, Koutou-ku, Tokyo 135-0064, \\ Japan: and ${ }^{c}$ Laboratory of Physical Chemistry for Drug Design, School of Pharmaceutical Sciences, Kitasato University; \\ 5-9-1 Shirokane, Minato-ku, Tokyo 108-8641, Japan. \\ Received August 5, 2003; accepted September 10, 2003; published online September 11, 2003
}

\begin{abstract}
Homology modeling was used to construct a model of the three-dimensional structure of pentachlorophenol 4-monooxygenase (PcpB). A PSI-BLAST homology search was initially performed to identify the 3D structure of proteins homologous with PcpB. The feasibility of modeled structures of PcpB was evaluated by Verify3D, which calculated structural compatibility scores based on 3D-1D profiles. The predicted structure of PcpB had an acceptable 3D-1D self-compatibility score, beyond the incorrect fold score threshold. A PcpB-pentachlorophenol (PCP) complex was then constructed utilizing the modeled PcpB structure. After energy minimization of the complex, and successive minimizations of the system that consisted of the complex and the water layer surrounding the complex, the molecular dynamics of the system were simulated. The active-site residues of PcpB were identified on the basis of the modeled structure, and PcpB mutants were then designed to change the active site residues, expressed, and purified by affinity chromatography. The mutant activity was compared with that of the wild-type to investigate the validity of the modeled structure. The experimental results suggested that Phe85, Tyr216, and Arg235 were relevant to enzyme activity, and that Tyr397 and Phe87 were important for stabilization of the structure of PcpB.
\end{abstract}

Key words pentachlorophenol 4-monooxygenase (PcpB); homology modeling; molecular dynamics; site-directed mutagenesis; bioremediation

Pentachlorophenol (PCP) is a polychlorinated aromatic compound that has been used as a pesticide and herbicide for many years, and its widespread use has caused contamination of soil and groundwater. ${ }^{1)}$ In addition, paper pulp mill bleach effluent contains large amounts of chlorinated phenols, including PCP. ${ }^{2)}$ PCP is listed as a Priority Pollutant by the Environmental Protection Agency because of its toxicity and widespread distribution in the environment. ${ }^{3)}$ Bioremediation of PCP contamination has therefore become an important focus of research.

Pentachlorophenol 4-monooxygenase (PcpB) has been purified from the PCP-degrading bacterium Sphingobium chlorophenolicum (formerly Sphingomonas chlorophenolica) strain ATCC9723. $\left.{ }^{4}{ }^{6}\right)$ This enzyme is a flavin monooxygenase that converts PCP to tetrachlorobenzoquinone (TCBQ) in the presence of oxygen and a reduced form of nicotinamide adenine dinucleotide phosphate (NADPH). ${ }^{7)}$ The nucleotide sequence of the $p c p B$ gene has already been determined and its recombinant protein has been produced, ${ }^{8,9)}$ but its three-dimensional (3D) structure has not yet been solved. In fact, little information is currently available regarding the structure and catalytic mechanism of action of this enzyme.

Computational chemistry and molecular modeling offer effective tools to study the mechanisms of biodegradation reactions at the molecular level, ${ }^{10-12)}$ and can provide guidelines for mutagenesis experiments. We have therefore used computer modeling for the construction of 3D-structures and the analysis of active-site residues in PcpB. PcpB was selected, first, because it appears to catalyze the rate-limiting step in the biodegradation of $\mathrm{PCP}^{3,13)}$ Thus, with a PcpB mutant it may be possible to degrade PCP more efficiently than with the wild-type. Second, PcpB is strikingly non-specific, unlike most flavin monooxygenases. It can turn over a variety of substituted phenols and can replace hydrogen, nitro, amino, and cyano groups, as well as halogens, with a hydroxyl group. ${ }^{14)}$ Consequently, it seems that PcpB can be altered to break down synthetic organic pollutants that natural microorganisms currently are unable to degrade.

In the present study, the 3D structure of PcpB was derived from homology modeling, molecular mechanics (MM) calculations, and molecular dynamics (MD) calculations, and the active-site residues of $\mathrm{PcpB}$ were analyzed. Protein expression and the activities of purified $\mathrm{PcpB}$ mutants, designed on the basis of the modeled structure, were compared with those of wild-type PcpB to investigate the model's validity.

\section{Experimental}

Homology Modeling of PcpB The amino acid sequence of PcpB was used as the search entry in the NR sequence database, using the positionspecific iterated basic local alignment search tool (PSI-BLAST), ${ }^{15)}$ iterating five times. The 3D structures of phenol hydroxylase $(\mathrm{PH})$ chain $\mathrm{D}$ (Protein Data Bank (PDB) ${ }^{16)}$ ID: 1FOH, PSI-BLAST E-value $\left.1 \times 10^{-59}\right)^{17)}$ and $p$-hydroxybenzoate hydroxylase (PHBH) (PDB ID: 1PBE, PSI-BLAST E-value $\left.2 \times 10^{-54}\right)^{18)}$ were extracted from the PDB and used as templates. The crystal structure of 'closed' PH (chain D) was selected from among four crystal structures, because the closed conformation is favorable for hydroxylation. The putative PcpB domains were determined by multiple sequence alignment using ClustalW ${ }^{19)}$ and visual inspection. The alignment is shown in Fig. 1. The structure of variable regions between domains was generated by finding suitable peptide segments in other proteins from the PDB. The conformations of complicated and 3D amino acid groups were revised using the rotational isomer library, and this 3D structure was finally optimized by the molecular mechanics method with the constant valence force field (CVFF). The homology modeling and energy optimizations of PcpB were carried out 
using Insight II Homology and Discover 3 modules (v2000, Accelrys Inc., San Diego, CA, U.S.A.) on a SGI workstation (Silicon Graphics Inc., Mountain View, CA, U.S.A.). The final PcpB homology model was evaluated by Verify3D Structure Evaluation Server. ${ }^{20)}$

Construction of the PcpB-PCP Complex The modeled structure of PcpB was superimposed on the 3D structure of PH (PDB ID: 1FOH), containing FAD and phenol (substrate). PCP was substituted for the substrate, and then docked manually into the binding site without significant conflict. The CVFF parameters of Discover 3 were used for the MM and MD simulations of the PcpB-PCP complex. Due to the lack of hydrogen atoms in the PDB structure, the initial positions of the hydrogen atoms were generated by the Modify/Hydrogens command in the Biopolymer module of the Insight II package. The energy-minimized complex was placed in a sphere consisting of 21992 TIP3P water molecules. ${ }^{21)}$ The radius of the sphere was $59 \AA$ and the $7-\AA$ outer layer was restrained (tethering, force constant 500) because of the prevention of water vaporization during $\mathrm{MM}$ and $\mathrm{MD}$ simulations. The 10 amino acid residues from the N-terminal were deleted, and the N-terminal was capped with an acetyl group so that the aqueous sphere could sufficiently hold the complex. Finally, the entire system was energy minimized until the value of final convergence was below 0.4. In the potential energy minimization, the steepest descent (SD) method was used for the early cycles, until the value of final convergence was below 8.0, and then the conjugate gradient (CG) method was used. The MD simulation of the energy-minimized system was run on a SGI Octane-workstation using Discover 3 modules in Insight II. This simulation was carried out at a constant temperature of $298 \mathrm{~K}$ and time step of $1 \mathrm{fs}$. The 600-ps MD calculation was performed. Every 10-ps structure, from 550 to $600 \mathrm{ps}$, was extracted from the MD trajectory file and the structures were then energy minimized under the same conditions as described above, with the exception of CG final convergence, which was 0.8 . The resultant structures of the PcpB-PCP complex were inspected and the putative active site residues of PcpB were identified. Secondary structure elements were calculated using the Kabsch-Sander method. ${ }^{22)}$

Bacterial Strains, Plasmids, and Culture Conditions S. chlorophenolicum ATCC39723 was purchased from Sumitomo Pharmaceuticals International Co. (SPI, Tokyo, Japan). The vector pET-19b (Novagen, Madison, WI, U.S.A.), which carries an N-terminal histidine tag sequence, followed by an enterokinase site and three cloning sites, was used for the cloning and expression of recombinant PcpB. The vector pKF18K (TaKaRa Shuzo, Kyoto, Japan) was used for construction of PcpB mutants. S. chlorophenolicum ATCC 39723 was cultured in mineral salt medium at $25^{\circ} \mathrm{C}^{3)}$ with shaking. Escherichia coli strain DH5 $\alpha$ (Toyobo, Osaka, Japan) was cultured in LuriaBertani (LB) medium ${ }^{23)}$ and E. coli strain BL21 (DE3) pLysS (Novagen) was cultured in Terrific Broth (TB $)^{23)}$ at $37^{\circ} \mathrm{C}$ with vigorous shaking. Media were solidified with $1.5 \%$ Bacto agar (Difco Laboratories, Detroit, MI, U.S.A.) and, where appropriate, supplemented with kanamycin or carbenicillin at final concentrations of 200 and $50 \mu \mathrm{g} / \mathrm{ml}$, respectively. Restriction enzymes were purchased from TaKaRa Shuzo. Primers for site-directed mutagenesis were purchased from Sigma Genosys Japan (Hokkaido, Japan).

Site-Directed Mutagenesis The pET-19b vector, described above, was digested with $B a m \mathrm{HI}$ and $X b a \mathrm{I}$. The obtained $p c p B$ fragment was ligated into the pKF18K vector. Site-directed mutagenesis of PcpB was performed with the pKF18K vector and a Mutan-Express Km kit (TaKaRa Shuzo). The provided protocol was followed with one exception; the kanamycin concentration was changed to $200 \mu \mathrm{g} / \mathrm{ml}$. All of the nucleotide sequences of mutants were confirmed by the dideoxy-chain termination method with an automated DNA sequencer (ABI PRISM 3700 genetic analyzer, Applied Biosystems, Foster City, CA, U.S.A.). The primers for site-directed mutagenesis were phosphorylated by T4 polynucleotide kinase (TakaRa Shuzo) and ATP prior to site-directed mutagenesis and had the following sequences (the underlined codons include the italicized substitutions that encoded the mutation): Phe85Ala (5'-GTCAGGAGCAACGGGGCGACGTTCAACTTCGAG-3'), Phe87Ala (5'-GCAACGGGTTCACGGCGAACTTCGAG-3'), Tyr216Ala (5'-GACGACTGGATTCACGCGTTCATCGGTCAGGAC-3'), Tyr216Phe (5'-CGACTGGATTCACT TC TTCATCGGTCAGG-3'), Phe223Ala (5'-GGACAAAGCGGTCTTCGTGACGAAGC-3'), Phe225Ala (5'-CAGGACAAATTCGTCGCGGTGAC-3'), Arg235Ala (5'-GCCGGGTTCCAATTAT $G C G$ GTGATTATCAGCGACC-3'), Arg235Glu (5'-CGGGTTCCAATTAT GAAGTGATTATCAGCGAC-3').

Mutants Tyr397Ala, Tyr397Phe, and Arg235Lys were constructed by the Long and Accurate PCR method with the same procedure as mentioned above for the Mutan-Express Km kit. These mutants were also phosphorylated and used as PCR primers with the selection primer of the kit. The sequences were: Arg235Lys (5'-GCCAAGGTCGCTGATAATCACCTTA-
TAATTGGAAC-3'), Tyr397Ala (5'-GCTGACGAGCTGGTCGCG CGCATTATAGG-3'), Tyr397Phe (5'-GCTGACGAGCTGGTCGCG $\underline{\text { GAAATTATA- }}$ GG-3').

PCR was performed, using these primers, for 20 cycles of denaturation at $95^{\circ} \mathrm{C}$ for $30 \mathrm{~s}$, annealing at $50^{\circ} \mathrm{C}$ for $30 \mathrm{~s}$, and extension at $68^{\circ} \mathrm{C}$ for $2 \mathrm{~min}$.

Enzyme Expression and Purification The modified pET-19b vectors, as constructed above, were transformed into E. coli BL21 (DE3) pLysS strain. The cells were allowed to grow in TB medium in the presence of carbenicillin $(200 \mu \mathrm{g} / \mathrm{ml})$ at $37^{\circ} \mathrm{C}$ until the optical density $\left(\mathrm{OD}_{600}\right)$ of the medium reached 0.6 . The expression of the desired protein was induced by adding isopropyl $\beta$-D-thiogalactopyranoside and carbenicillin to final concentrations of $100 \mu \mathrm{M}$ and $500 \mu \mathrm{M}$, respectively. The cells were incubated at $20^{\circ} \mathrm{C}$ overnight, and all purification steps were performed at $4{ }^{\circ} \mathrm{C}$. All buffers contained $20 \mathrm{~mm} \beta$-mercaptoethanol and $15 \%$ (v/v) glycerol. The cells were collected by centrifugation, suspended in $50 \mathrm{~mm} 2$-amino-2-(hydroxymethyl)-1,3-propanediol (Tris) $-\mathrm{HCl}(\mathrm{pH} 8.0)$ and $0.09 \%$ (v/v) Triton $\mathrm{X}-100$, and broken by sonication (TOMY Ultrasonic disruptor UD-201, TOMY Digital Biology, Tokyo, Japan). The suspension was centrifuged and the soluble fraction of the enzyme was mixed with the same amount of bind buffer containing $800 \mathrm{~mm} \mathrm{NaCl}$ and $1.6 \mathrm{~mm}$ imidazole. The solution was loaded into an Econo Column $(1.5 \times 15 \mathrm{~cm})$ (BioRad, Hercules, CA, U.S.A.) packed with Ni-NTA agarose (Qiagen K. K., Tokyo, Japan) equilibrated in buffer containing $50 \mathrm{~mm}$ Tris- $\mathrm{HCl}(\mathrm{pH} 8.0$ ) and $400 \mathrm{~mm} \mathrm{NaCl}$. The solution was treated with two kinds of wash buffer. Buffer 1 contained $50 \mathrm{~mm}$ Tris- $\mathrm{HCl}\left(\mathrm{pH} 8.0\right.$ ), $50 \mathrm{~mm} \mathrm{NaCl}, 0.8 \mathrm{~mm}$ imidazole, $5 \mathrm{mM} \mathrm{MgCl}_{2}$, and $0.1 \mathrm{~mm}$ EDTA-Na (pH 8.0). Buffer 2 was the same as buffer 1, except that the concentration of imidazole was $40 \mathrm{~mm}$ instead of $0.8 \mathrm{~mm}$. PcpB activity was then eluted with the elution buffer, which was the same as the wash buffers except that the imidazole concentration was $200 \mathrm{~mm}$. The eluate was concentrated by ultrafiltration (Amicon Centriprep YM-30, Millipore, Billerica, MA, U.S.A.), loaded onto NAP10 columns (Amersham Biosciences, Upsalla, Sweden), and equilibrated in $50 \mathrm{~mm}$ Tris $-\mathrm{HCl}(\mathrm{pH} 8.0)$ and $15 \%$ $(\mathrm{v} / \mathrm{v})$ glycerol. The imidazole was removed from the buffer using the NAP 10 column. The eluate was again concentrated by ultrafiltration (Amicon Centricon YM-30, Millipore) and stored at $-80{ }^{\circ} \mathrm{C}$. In the case of Y397 mutants, the enzyme was purified and concentrated in the presence of $25 \%$ glycerol, to stabilize the enzyme.

Enzyme purity and the molecular weights of the subunits were determined by sodium dodecyl sulfate-polyacrylamide gel electrophoresis (SDS-PAGE), with $5-20 \%$ gradient gels (PAGEL NPG520L, ATTO, Tokyo, Japan) and molecular weight markers (Daiichi III, Daiichi Pure Chemicals, Tokyo, Japan). The proteins were stained with Bio-Safe CBB G-250 Stain (BioRad). The protein concentration was determined by the method of Bradford, ${ }^{24)}$ with bovine serum albumin (BSA) as the standard.

Comparison of the Activity of PcpB and PcpB Mutants The activity of $\mathrm{PcpB}$ and its mutants was assayed by measuring the production of tetrachlorohydroquinone (TCHQ), which is probably produced by NADPH-reduction of the reaction product (TCBQ) of PCP and PcpB. ${ }^{7)}$ A 200- $\mu$ l enzyme solution was made from PcpB $(124.2 \mu \mathrm{g}, 10 \mu \mathrm{M}), 25 \%$ glycerol $(\mathrm{v} / \mathrm{v})$, and $10 \mathrm{mg} / \mathrm{ml}$ BSA (Fraction V, crystalline, Calbiochem-Novabiochem Co., La Jolla, CA, U.S.A.), because of enzyme stability. The assay system contained $80 \mu$ l of $100 \mathrm{~mm}$ potassium phosphate (KPi) buffer ( $\mathrm{pH} 7.0$ ), $5 \mu \mathrm{l}$ of $2 \mathrm{~mm}$ NADPH, $1 \mu \mathrm{l}$ of $2 \mathrm{~mm}$ PCP, and $5 \mu \mathrm{l}$ of enzyme solution in a volume of $100 \mu \mathrm{l}$. The PCP degradation reaction by PcpB and its mutants was carried out for $90 \mathrm{~s}$ at a constant temperature of $25^{\circ} \mathrm{C}$. The extent of reaction by wild-type PcpB was approximately $5 \%$ under these conditions. The reaction mixtures were acidified with $20 \mu \mathrm{l}$ of $2 \mathrm{~mol} / \mathrm{l} \mathrm{HCl}$, and extracted 3 times with $100 \mu 1$ of ethyl acetate after the reaction. The ethyl acetate was taken up into glass inserts in screw-capped vials for GC-MS. Aliquots of ethyl acetate solution containing $200 \mathrm{ng}$ of pentachlorothioanisole (PCTA) used as an external standard were also taken up into the glass inserts. Following evaporation of the ethyl acetate under a stream of nitrogen gas, the residue was dissolved in $100 \mu$ l of a $2: 1$ solution of pyridine and acetic anhydride, for acetylation of PCP and TCHQ, and allowed to stand at room temperature for several hours. The production of acetylated TCHQ was analyzed by GC-MS. Activities of PcpB mutants were calculated relative to that of wild-type. Experiments were repeated 4 times.

Analytical Methods GC-MS analysis was performed with a model HP 6890 series GC system and 5973 Mass Selective Detector system (Agilent Technologies, Palo Alto, CA, U.S.A.) fitted with a fused silica chemically bonded capillary column (DB- $5 \mathrm{~W}$; inside diameter, $0.25 \mathrm{~mm}$; length, $30 \mathrm{~m}$; film thickness, $0.25 \mu \mathrm{m}$ : J\&W Scientific, Inc., Folsom, CA, U.S.A.). Each 1$\mu 1$ sample was injected into the column at $130^{\circ} \mathrm{C}$ in splitless mode, using an HP 7863 series injector and auto sampler. After $1 \mathrm{~min}$ at $130^{\circ} \mathrm{C}$, the column 


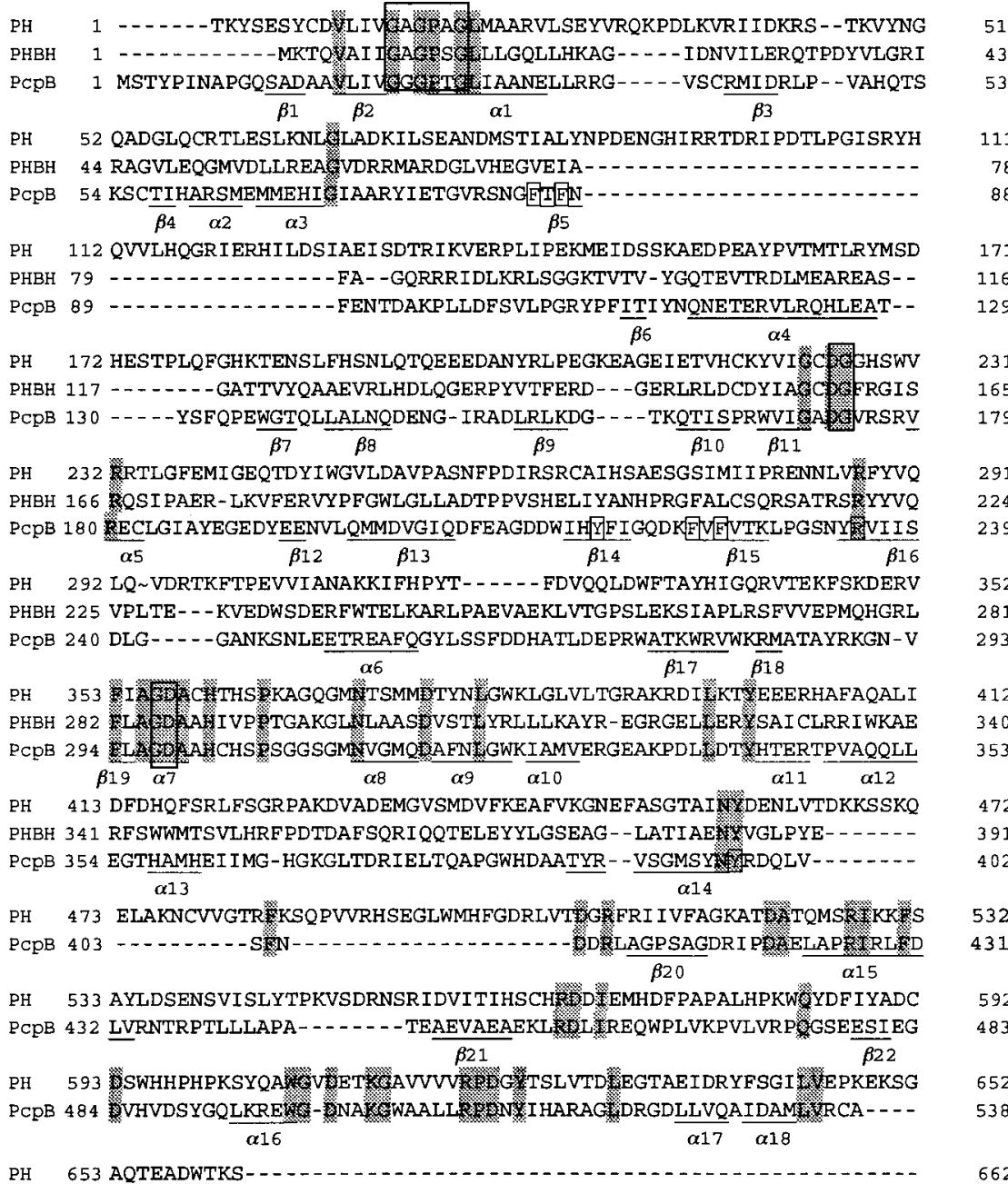

Fig. 1. The Protein Sequence Alignment of PcpB and Two Types of Hydroxylase Used in Homology Modeling

Sequence alignment was performed using ClustalW and further modified manually. The sequences used for the alignment were phenol hydroxylase (PH) (PDB ID: 1FOH) and $p$-hydroxybenzoate hydroxylase (PHBH) (PDB ID: 1PBE). Conserved residues are shaded. The residues mutated in the experimental section are in squares. In PDB data of 1FOH, residues from Ala294 to Arg301 have been deleted, as indicated by ' $\sim$ '. The three consensus sequences (GXGXXG, DG, GD) in the flavoprotein hydroxylases are boxed. The positions of the secondary elements in PcpB are indicated by underlined sequences. The secondary structure elements of PcpB are numbered according to the predictions of the Kabsch-Sander method.

temperature was increased by $10^{\circ} \mathrm{C} / \mathrm{min}$ to $250^{\circ} \mathrm{C}$, and held at $250^{\circ} \mathrm{C}$ for $4 \mathrm{~min}$. The amounts of PCTA were calculated based on the peak area, determined by the selected ion monitoring method. The amounts of acetylated PCP and TCHQ were calculated based on the calibration curves of these compounds relative to PCTA. The selected $\mathrm{m} / \mathrm{z}$ of these compounds were as follows: PCTA 246, 296; PCP 266, 308; and TCHQ 248, 290, 332.

Chemicals PCP, PCTA, and ethyl acetate were purchased from Wako Pure Chemical Ind., Ltd. (Osaka, Japan). These chemicals were of residual pesticide analysis grade, and the purities of PCP and PCTA were more than $99 \%$. All other chemicals were of analytical grade and of the highest purity available.

\section{Results and Discussions}

Construction of 3D Structure of PcpB by Homology Modeling The PSI-BLAST homology search ${ }^{15)}$ was initially performed to identify the $3 \mathrm{D}$ structures of proteins homologous to $\mathrm{PcpB}$, because no template proteins for PcpB were found on the BLAST search. PH and PHBH were selected as templates, with significant E-values, for construction of the PcpB homology model. PcpB showed 21.0\% and $16.5 \%$ sequence identity to $\mathrm{PH}$ and $\mathrm{PHBH}$, respectively. PSIBLAST methods have been well benchmarked, with low sequence similarity (less than $30 \%)$, ${ }^{25-27)}$ and both enzymes are well-studied flavoproteins that catalyze hydroxylation of the ortho position to the hydroxyl group of a substrate (phenol or $p$-hydroxybenzoate) in the presence of oxygen and NADPH. The same substances, FAD, NADPH, and oxygen, were also used in PcpB. Moreover, the three consensus sequences in the hydroxylases were also conserved in $\mathrm{PcpB}$ (Fig. 1). ${ }^{28)}$ Thus, it is likely that PcpB and these two proteins have similar enzymatic functions. The construction of a structural model for PcpB was therefore attempted with the Homology module of Insight II, as described in the Experimental section. The feasibility of the modeled structure of PcpB was evaluated by Verify3D, which calculated the structural compatibility score, based on the 3D-1D profile. ${ }^{20)}$ The predicted structure of PcpB had an acceptable 3D-1D selfcompatibility score (159.05), beyond the incorrect fold score threshold (111.01).

PcpB-PCP Complex and the Prediction of Active Site Residues The PcpB-PCP complex was constructed utilizing the above PcpB structure, as described in the Experimental section. After energy minimization of the complex, and successive minimization of the system that consisted of the 
(a)

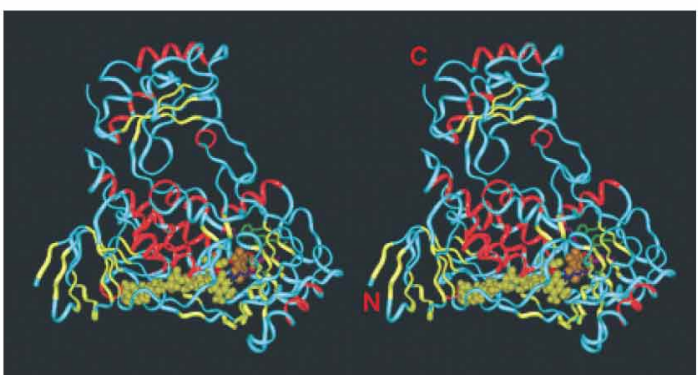

(b)

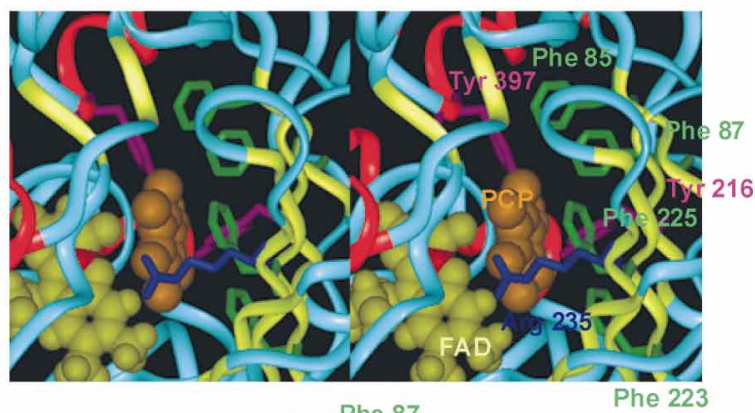

(c)

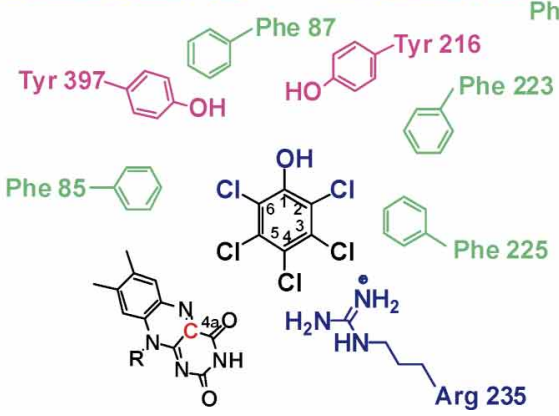

Fig. 2. The 3D Structure of PcpB, Constructed by Molecular Modeling

(a) Stereo view of the entire structure of PcpB. The color of the ribbon corresponds to the secondary structure elements calculated by the Kabsch-Sander method. The regions of red, yellow, and light blue refer to $\alpha$-helix, $\beta$-sheet, and random coil, respectively. The N-terminal and C-terminal are shown in capital letters. (b) Stereo view of the active site of PcpB. Important residues, predicted from inspection, are shown and distinguished with different colors: Tyrosine, phenylalanine, and arginine are shown in pink, light green, and blue, respectively. PCP and FAD are shown in orange and yellow CPK models, respectively. The red atom in FAD shows the $\mathrm{C}_{4 \mathrm{a}}$ atom to which molecular oxygen is likely added. (c) A schematic of the active site of PcpB shown in (b). The blue atoms in PCP are important for PcpB recognition.

complex and the water layer surrounding the complex, MD simulation of the system was performed. The resultant structure of the PcpB-PCP complex is shown in Fig. 2a.

From inspection of the active site residues in $\mathrm{PcpB}, 7$ residues seemed to be in direct contact with either the substrate molecule or with each other, as shown in Fig. $2 b$ and 2c. Tyr216 and Tyr397 were selected because these tyrosines had the potential to make hydrogen bonds between the enzyme and the hydroxyl group of the substrate in both $\mathrm{PH}$ $\left(\right.$ Tyr289) ${ }^{17)}$ and PHBH (Tyr201). ${ }^{18)}$ The residues Tyr216 and Tyr397 were conserved in the template enzyme (Fig. 1). Moreover, the residues Tyr201 and Tyr385 in PHBH, which corresponded to Tyr216 and Tyr397 in PcpB, respectively, played important roles in recognition and substrate specificity of $\mathrm{PHBH}{ }^{29)}$ Arg235 was also conserved, and its positively charged guanidinium group seemed to stabilize the chloride ion released from PCP during the enzymatic reaction. To our knowledge, such a role of arginine in other enzymes has not been reported, but the chloride-arginine interaction has been reported in some proteins such as halorhodopsin ${ }^{30)}$ and deoxyhemoglobin Rothschild. ${ }^{31)}$

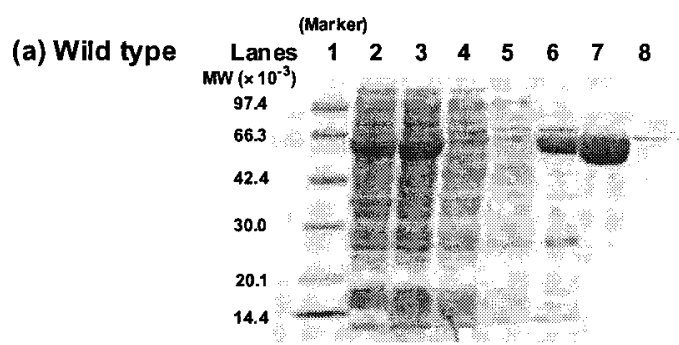

(b) Tyr397Ala

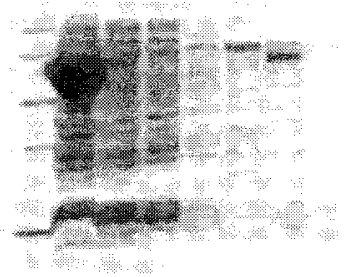

(d) Phe87Ala

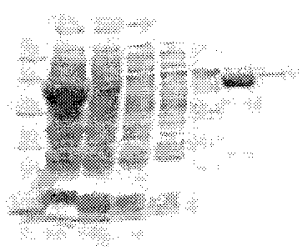

(c) Tyr397Phe

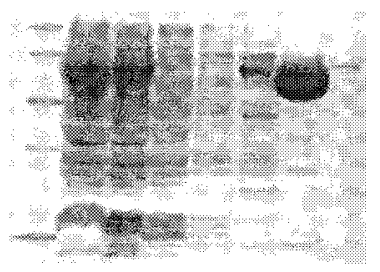

(e) Arg235Lys

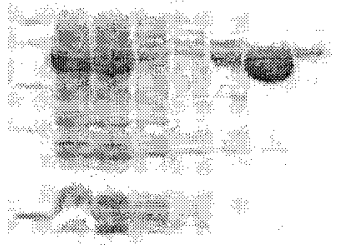

Fig. 3. Purification Steps of Recombinant PcpB (a) and Its Mutants (be), Traced by SDS-PAGE

The type of mutant is shown above each figure. Lanes: 1 , molecular size markers; 2 , cell suspension after sonication; 3 , supernatant after centrifugation of the cell suspension; 4, the fraction after the supernatant was passed through the column; 5 , the fraction of wash 1 buffer; 6 , the fraction of wash 2 buffer; 7 , the fraction of elution1 buffer; 8 , the fraction of elution 2 buffer.

Phe225 possibly made a $\pi-\pi$ interaction with PCP. The phenyl ring edge of Phe87 seemed to interact with the $\pi$ face of Tyr397. Finally, Phe85 and Phe223 seemed to be involved in recognition of the ortho chloride of PCP, and was therefore selected, because PcpB could convert the substrate for both dichlorinated and trichlorinated phenols as long as one of the ortho positions was chlorinated. ${ }^{14)}$

Expression and Purification of the PcpB Mutants Recombinant PcpBs with mutations in the seven residues were expressed in E. coli and purified by affinity chromatography. The process of purification of PcpB and some of its mutants is shown in Fig. 3. The purified protein was manifested as a major band on SDS-PAGE, with an apparent molecular mass of approximately 60000, as reported previously. ${ }^{5)}$ PcpB was readily purified because the enzyme moved to the soluble fraction (Fig. 3a, lane 3 and lane 7). This result is consistent with those of a previous report, which indicated that PcpB was translocated from the cytoplasm, via the inner membrane, to the periplasm of $S$. chlorophenolicum, and that the majority of PcpB was soluble at all times. ${ }^{32}$ Most PcpB mutants, such as Tyr397Phe and Arg235Lys, showed similar behavior (Figs. 3c, e). Only small amounts of Tyr397Ala and Phe87Ala mutants moved to the soluble fraction, however, presumably due to the structural change of the protein (Figs. $3 \mathrm{~b}, \mathrm{~d})$. The results of SDS-PAGE for other mutants are shown in the supporting information (Fig. 5).

Comparison of the Activities of $\mathrm{PcpB}$ and $\mathrm{PcpB} \mathrm{Mu}-$ 


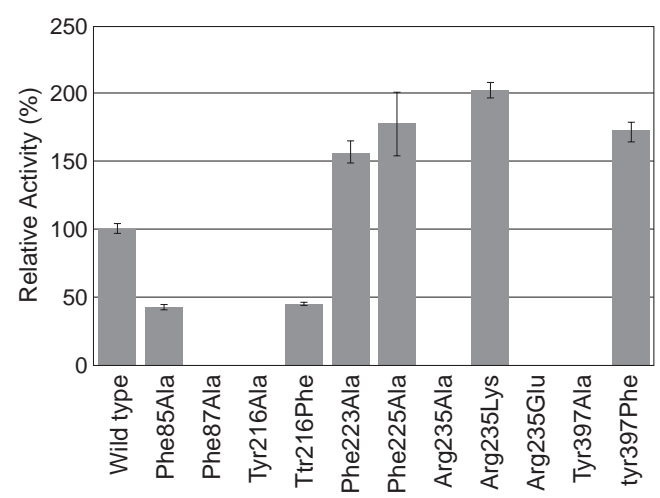

Fig. 4. Relative Activities of the PcpB Mutant in Comparison with That of Wild-Type PcpB

The amount of TCHQ made from PCP with wild-type PcpB (Wild type) was used as a standard. The mutant type is shown below the histogram. Data are shown as means \pm standard deviation, with 4 replicates per treatment.

tants The amounts of TCHQ produced by $\mathrm{PcpB}$ and NADPH via TCBQ were subsequently analyzed by GC-MS, and the activities of PcpB mutants were calculated relative to that of the wild type. The results are shown in Fig. 4. Replacement of either Tyr216 or Tyr397 with alanine greatly decreased the activity. Replacement of either Tyr216 or Tyr397 with phenylalanine, however, decreased activity by $60 \%$ in Tyr216Phe and increased activity by $60 \%$ in Tyr397Phe. Thus, Tyr216 appears to be more important for the mechanism of action of PCP than Tyr397.

Replacement of Arg235 with alanine and glutamic acid markedly decreased the activity, but replacement by lysine increased activity. The positive charge of the guanidinium group in arginine could be important for the reaction of PcpB. The proposed role of Arg235 for the stabilization of the released chloride ion is interesting and specific to PcpB, because such a function was not obvious in either template enzyme, even though the sequence was conserved (Fig. 1). Thus, the function of Arg235 might come from the evolution of a metabolic pathway of the template enzymes. Replacement of Phe87 with alanine greatly decreased enzyme activity. As shown in Figs. 2b and d, the benzene rings of Phe87 and Tyr397 likely play an important role in stabilizing the active site of PcpB. Interestingly, establishing the 3D-structure revealed that Phe87 is important in stabilizing the active site of PcpB (Figs. 2c, 3d). Moreover, replacement of Phe85 with alanine decreased activity by $60 \%$, suggesting the importance of the Phe 85 benzene ring.

The reaction mechanism of $\mathrm{PcpB}$ is not obvious, but the reaction likely proceeds via electrophilic attack of an intermediate hydroperoxide form of the flavin cofactor $\left(\mathrm{C}_{4 \mathrm{a}}\right.$-hydroperoxyflavin) on the $\mathrm{C}_{4}$ substrate as in $\mathrm{PHBH}$ and $\mathrm{PH}$, which have been well studied. ${ }^{33-35)}$ Thus, the distance between the $\mathrm{C}_{4 \mathrm{a}}$ atom of FAD and the substrate $\mathrm{C}_{4}$ atom in the modeled structure of PcpB was compared to the distance between the $\mathrm{C}_{4 \mathrm{a}}$ atom of FAD and the substrate $\mathrm{C}_{2}$ atom in the crystal structures of $\mathrm{PH}$ and $\mathrm{PHBH}$. The result was indicative of the propriety of the modeled structure, because the distance in PcpB, $4.73 \AA$, was between those of PHBH, $4.37 \AA$, and $\mathrm{PH}, 5.37 \AA$. Moreover, the flavin cofactors, present in the model structure of $\mathrm{PcpB}$ or in the crystal structure of $\mathrm{PH}$ and $\mathrm{PHBH}$, were modified to the active $\mathrm{C}_{4 \mathrm{a}}$-hydroperoxyflavin intermediate using the coordinates reported previously ${ }^{36)}$ on the

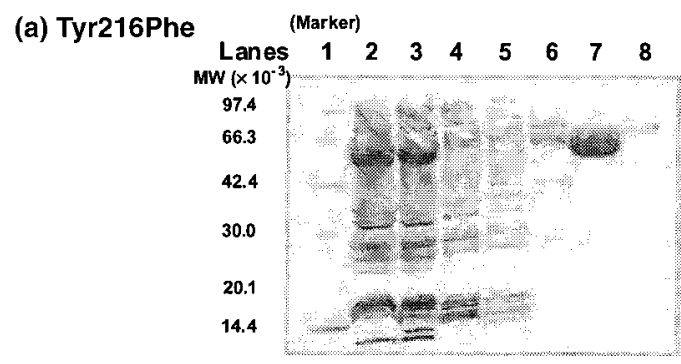

(b) Phe223Ala

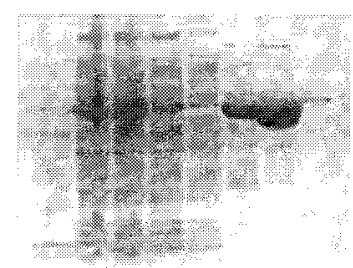

(d) Arg235Ala

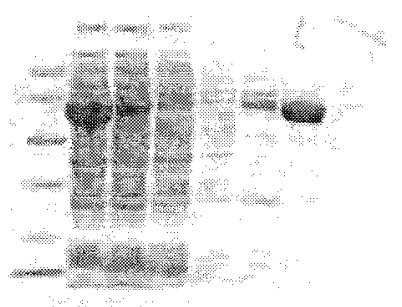

(c) Phe225Ala

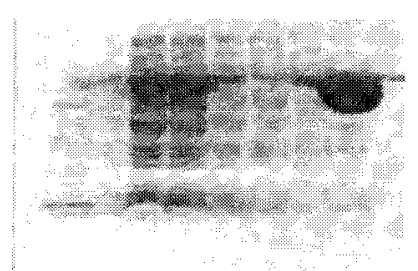

(e) Arg235Glu

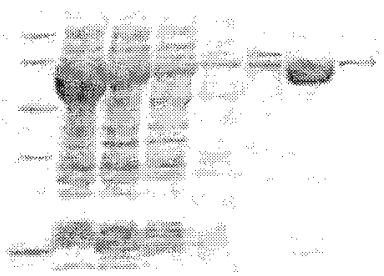

Fig. 5. Purification Steps of PcpB Mutants Traced by SDS-PAGE

(a) Tyr216Phe, (b) Phe223Ala, (c) Phe225Ala, (d) Arg235Ala, and (e) Arg235Glu. Conditions are as same as that of Fig. 3.

basis of molecular modeling studies. Schreuder et al. ${ }^{37)}$ suggested in the previous report that the almost ideal position of the distal oxygen, at an approximate angle of $60^{\circ}$ with the plane of the aromatic ring of the substrate, is certainly important in enhancing the reaction rate. Thus, the distance between the distal oxygen from FAD $\left(\mathrm{O}_{\mathrm{d}}\right)$ and the substrate carbon atom $\left(\mathrm{C}_{\mathrm{r}}\right)$, which is attacked to the $\mathrm{O}_{\mathrm{d}}$ atom, and the angle formed by the line $\mathrm{O}_{\mathrm{d}}-\mathrm{C}_{\mathrm{r}}$ with the plane of the aromatic ring were examined. The distance $\mathrm{O}_{\mathrm{d}}-\mathrm{C}_{4}$ and angle formed by the line $\mathrm{O}_{\mathrm{d}}-\mathrm{C}_{4}$ with the plane of the aromatic ring were $3.5 \AA$ and $81^{\circ}$, respectively in the case of PcpB. This result was comparable with those of $\mathrm{PH}, 3.2 \AA$ and $74^{\circ}$.

The model did not explain all the results of site-directed mutagenesis; for example, the increased activities of Phe223Ala and Phe225Ala mutants relative to the wild-type were not explained. The 3D-structure of PcpB optimized by further MD simulation or partial modification might be able to account for the experimental result. The measurement of kinetic parameters, such as $K_{\mathrm{m}}$ and $k_{\text {cat }}$, and the analysis of structural change induced by site-directed mutagenesis are still required. This study provided the first analysis of the $3 \mathrm{D}$ structure of PcpB. PcpB is an attractive enzyme for bioremediation, because PCP should be a model compound of recalcitrant polychlorinated aromatic compounds, such as PCBs and dioxin. Thus, it is very important that PcpB be designed for improved degradation and substrate specificity.

Acknowledgments We are grateful to the Research Resources Center at the Riken Brain Science Institute for DNA sequencing. We thank Drs. Noriaki Okimoto from RIKEN in Japan and Jiri Damborsky from the National Center for Biomolecular Research in the Czech Republic for helpful discus- 
sion. This work was supported by The Ministry of Education, Culture, Sports, Science, and Technology (MEXT) through a Grant-in-Aid for Young Scientists (B) (grant no. 14760067).

\section{References}

1) McAllister K. A., Lee H., Trevors J. T., Biodegradation, 7, $1-40$ (1996).

2) Thakur I. S., Verma P. K., Upadhaya K. C., Biochem. Biophys. Res. Commun., 286, 109-113 (2001).

3) McCarthy D. L., Claude A. A., Copley S. D., Appl. Environ. Microbiol., 63, 1883-1888 (1997).

4) Saber D. L., Crawford R. L., Appl. Environ. Microbiol., 50, 1512 1518 (1985).

5) Xun L., Orser C. S., J. Bacteriol., 173, 4447-4453 (1991).

6) Takeuchi M., Hamana K., Hiraishi A., Int. J. Syst. Evol. Microbiol., 51, 1405-1417 (2001).

7) Dai M. H., Rogers J. B., Warner J. R., Copley, S. D., J. Bacteriol., 185, $302-310(2003)$

8) Orser C. S., Lange C. C., Xun L., Zahrt T. C., Schneider B. J., J. Bacteriol., 175, 411-416 (1993)

9) Wang H., Tiirola M. A., Puhakka, J. A., Kulomaa, M. S., Biochem. Biophys. Res. Commun., 289, 161-166 (2001).

10) Bohac M., Nagata Y., Prokop Z., Prokop M., Monincova M., Tsuda M., Koca J., Damborsky J., Biochemistry, 41, 14272-14280 (2002).

11) Ridder L., Mulholland A. J., Rietjens I. M. C. M., Vervoort J., J. Am. Chem. Soc., 122, 8728-8738 (2000).

12) Ridder L., Palfey B. A., Vervoort J., Rietjens I. M. C. M., FEBS Lett., 478, 197-201 (2000).

13) Copley S. D., TIBS, 25, 261-265 (2000).

14) Xun L., Topp E., Orser C. S., J. Bacteriol., 174, 2898-2902 (1992).

15) Altschul S. F., Madden T. L., Schaffer A. A., Zhang J., Zhang Z., Miller W., Lipman D. J., Nucleic Acids Res., 25, 3389-3402 (1997).

16) Berman H. M., Westbrook J., Feng Z., Gilliland G., Bhat T. N., Weissig H., Shindyalov I. N., Bourne P. E., Nucleic Acids Res., 28, 235242 (2000).

17) Enroth C., Neujahr H., Schneider G., Lindqvist Y., Structure, 6, 605617 (1998).
18) Schreuder H. A., Prick P. A. J., Wierenga R. K., Vriend G., Wilson K. S., Hol W. G. J., Drenth J., J. Mol. Biol., 208, 679-696 (1989).

19) Thompson J. D., Higgins D. G., Gibson T. J., Nucleic Acids Res., 22, 4673-4680 (1994).

20) Luthy R., Bowie J. U., Eisenberg D., Nature (London), 356, 83-85 (1992).

21) Jorgensen W. L., Chandrasekhar J., Madura J. D., J. Chem. Phys., 79, 926-935 (1983).

22) Kabsch W., Sander C., Biopolymers, 22, 2577-2637 (1983).

23) Sambrook J., Fritsch E. F., Maniatis T., "Molecular Cloning: A Laboratory Manual," 2nd ed., Cold Spring Harbor Laboratory Press, New York, 1989.

24) Bradford M. M., Anal. Biochem., 72, 248-254 (1976).

25) Park J., Karplus K., Barret C., Hughey R., Haussler D., Hubbard T., Chothia C., J. Mol. Biol., 284, 1201-1210 (1998).

26) Salamov A. A., Suwa M., Orengo C. A., Swindells M. B., Protein Sci., 8, $771-777$ (1999)

27) Friedberg I., Kaplan T., Margalit H., Protein Sci., 9, 2278-2284 (2000).

28) Eppink M. H. M., Schreuder H. A., van Berkel W. J. H., Protein. Sci., 6, 2454-2458 (1997)

29) van der Bolt F. J. T., van den Heuvel R. H. H., Vervoort J., van Berkel W. J. H., Biochemistry, 36, 14192-14201 (1997).

30) Braiman M. S., Walter T. J., Briercheck D. M., Biochemistry, 33, 1629-1635 (1994).

31) Kavanaugh J. S., Rogers P. H., Case D. A., Arnone A., Biochemistry, 31, 4111-4121 (1992).

32) Wang H., Marjomaki V., Ovod V., Kulomaa M. S., Biochem. Biophys. Res. Commun., 299, 703-709 (2002)

33) Husain M., Entsch B., Ballou D. P., Massey V., Chapman P. J., J. Biol. Chem., 255, 4189-4197 (1980).

34) Maeda-Yorita K., Massey V., J. Biol. Chem., 268, 4134 -4144 (1993).

35) Entsch B., Berkel W. J. H., FASEB J., 9, 476-483 (1995).

36) Ridder L., Mullholland A. J., Rietjens I. M. C. M., Vervoort J., J. Mol. Graph. Mod., 17, 163-175 (1999).

37) Schreuder H. A., Hol W. G. J., Drenth J., Biochemistry, 29, 31013108 (1990). 